\title{
Experimental Test on Gfrp Gratings for Mechanical Properties and Chemical Resistance
}

\author{
B. Devender ${ }^{1}$, A.Purushotham ${ }^{2} \&$ O.Vasudeva Reddy ${ }^{3}$ \\ 1 [M.Tech (CAD/CAM) student, Department of mechanical engineering, Sree nidhi Institute of \\ Science and Technology (Autonomous), Hyderabad, A.P, India, \\ 2 [Supervisor, Professor, Department of mechanical engineering, Sree nidhi institute of science\& \\ technology, Hyderabad, A.P, India] \\ 3 [Managing Director, Advanced Millennium Materials, Phase-1, Cherlapally, Hyderabad, (AP) India]
}

\begin{abstract}
Fiberglass reinforced plastic (FRP) grating is manufactured by combining a resin and fiberglass. That makes it a composite material. Fiberglass grating does not corrode like steel gratings. They are used in corrosive environments. Fibre glass grating is formed using continuous interwoven glass fibres as reinforcement material and resin as the matrix with continuous solidification through a mould system. The uniform construction provides excellent bi-directional mechanical properties. It is beneficial to all industries whether it be chemical, pharmaceutical, offshore or leisure to name just a few with many applications like platforms, floorings, walkways, trench covers, ramps and cable trough covers. Fibreglass grating is exceptionally slip resistant and the gritted and concave anti-slip grating offers excellent traction in wet and icy conditions. In this work, molded fibre glass gratings are manufactured and subjected to tensile test, chemical resistance, water absorption, flexural test, load bearing test.
\end{abstract}

Keywords: Molded fibre glass gratings, glass fibers, tensile strength, flexural strength mechanical properties, and chemical resistance.

\section{Introduction}

A grating is any regularly spaced collection of essentially identical, parallel, elongated elements. Gratings usually consist of a single set of elongated elements, but can consist of two sets, in which case the second set is usually perpendicular to the first (as illustrated). When the two sets are perpendicular, this is also known as a grid or a mesh. Fiberglass grating is an integral construction single piece fiberglass roving reinforced with Isopthalic resin typically composed of $60-65 \%$ resin by weight and immensely strong continuous glass fibers manufactured by a specially designed process that provides a property of good mechanical and corrosion resistance. These are safe \& ideal for chemically corrosive environment having long maintenance free life.

Advantages of fibre glass grating are:

- High corrosion resistance level

- Electric insulation

- Durability therefore economical and available in anti skid

- Fire retardant and ultraviolet protection types.

\section{Material And Manufacturing Method}

The glass fiber rovings used for manufacturing of the gratings was supplied by Owens Corning India Ltd, with the designation 399. The matrix material was Isopthalic resin Polyester based on Isopthalic / superior mixed glycol is a high quality, medium viscosity, medium reactive specially formulated for processes like Filament winding, spray up/hand layup. It exhibits good chemical resistance and possess superior mechanical and thermal properties. The resin has exceptionally good hydraulic resistance making it an ideal choice for coating / laminating applications. The ISO resin forms strong bond with fiber glass rovings and depicts good wet out characteristics due to good weathering properties and retention of glass it is suitable for corrosion resistant applications.

\section{Manufacturing Of Frp Gratings}

FRP gratings are processed in two different manufacturing methods. Generally gratings are classified according to type of manufacturing process. They are: 
1. Molded FRP grating

2. Pultruded FRP grating

\section{Molded Fibre Glass Grating Manufacturing Process:}

A grating is any regularly spaced collection of essentially identical, parallel, elongated elements. Gratings usually consist of a single set of elongated elements, but can consist of two sets, in which case the second set is usually perpendicular to the first (as illustrated). When the two sets are perpendicular, this is also known as a grid or a mesh. Fiberglass grating is an integral construction single piece fiberglass roving reinforced with Isopthalic resin typically composed of $60-65 \%$ resin by weight and immensely strong continuous glass fibers manufactured by a specially designed process that provides a property of good mechanical and corrosion resistance. These are safe \& ideal for chemically corrosive environment having long maintenance free life.

Molded grating is manufactured in an open, heated mold that resembles a large waffle iron. Continuous glass fibres are placed in the mold in alternating layers and thoroughly wetted out with resin. This continuous process produces an integral, one-piece construction, which offers excellent corrosion resistance as well as bi-directional strength. When the weaving process is completed, the mold is heated to cure the panel. If the grating is to have embedded grit, the mold will receive the grit at this time before the part is cured. After curing, the part is extracted from the mold. The standard part would have a meniscus (concave) top surface for slip resistance. Should a standard grit surface be specified, the grit would be bonded to the top of the completed grating panel as a secondary operation.

Liquid resin and continuous fiberglass roving's systematically laid in a mold, layer after layer manually, to produce the desired thickness and panel dimensions. The finished molds are set aside for a predetermined time to allow the panel to cure. The panel is then ejected from the mold. The molds are cleaned and prepare for the process to begin again. The one piece interwoven mesh construction of molded fiberglass grating produces a product with optimal corrosion resistance and bi-directional strength. Since the fiberglass grating is "cast" in one piece, there is no mechanical joint between bearing bars, unlike pultruded grating where the glued joints can give way in heavy continuous traffic or after prolonged service. The high percentage of resin $(65 \%)$ in molded fiberglass grating offers superior corrosion resistance and optimal impact resistance. Molded fiberglass gratings with a square mesh pattern offer increased load capacity and panel utilization due to its bi-directional trait.

Numerous cut-outs for piping, valve access, and column penetrations can be made without the need for additional structural framing. Being of one-piece construction, the fiberglass grating performs as a plate, distributing loads throughout the fiberglass grating section and around cut-outs. Cutting access holes in the molded fiberglass grating does not weaken the panel and does not generally require additional or costly supports (unlike pultruded fiberglass grating that will require additional supports at cutouts)

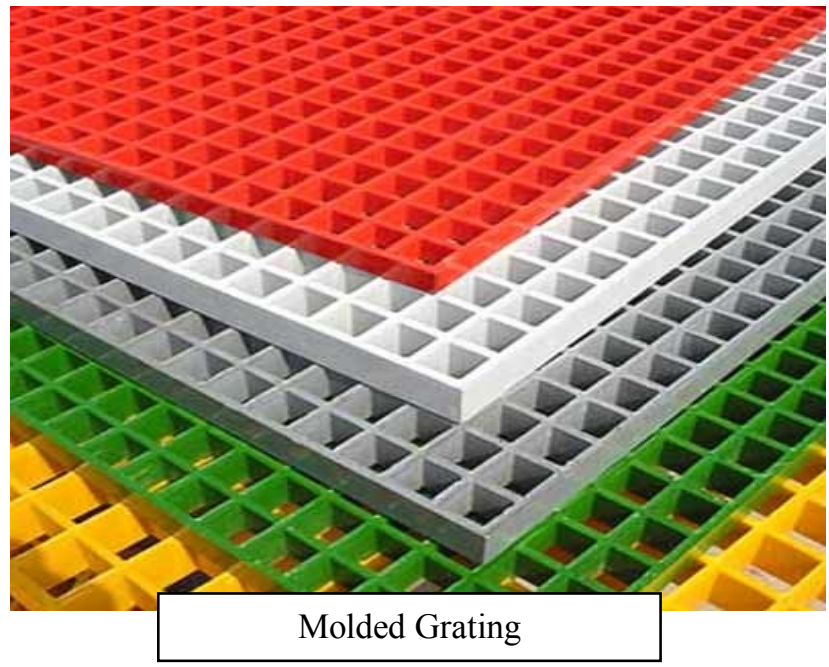

\section{Pultruded Grating Manufacturing Process:}

The pultrusion process utilized in the manufacturing of pultruded fiberglass grating and structural shapes is a continuous and automated process where continuous fiberglass rovings and mat are pulled through guides, a resin 
bath and pre-formers, then wrapped with a synthetic veil before being pulled through a heated die. The individual components of pultruded fiberglass grating - load bars and each piece of the two piece tie-bar are pultruded separately.

Load bars are then cut to specified lengths as they exit the machine. They are drilled at 6" or 12" centers for tie-bar insertion. Once the bars have been drilled they are spaced proportionately for the width of the panel at $3 \mathrm{ft}, 4 \mathrm{ft}$ or 5 $\mathrm{ft}$. The two piece locking tie bar assemblies are then inserted creating multiple bonded intersections of load bars and tie bars, thus providing the security of both a mechanically locked and bonded connection. The final step in the process is to seal the cross bars and holes with corrosion resistant epoxy resin.

Pultruded fibre glass grating is unidirectional in strength and provides increased load capacity and very good levels of corrosion resistance. The components (bearing bars and cross bars) which are pulled by machine provide a higher glass to resin ratio content (65\% glass to $35 \%$ resin) which gives it superior load capacity, yet reduces the corrosion resistance when compared to molded fiberglass grating.

Assembled from components, pultruded fiberglass grating can offer a wide variety of panel sizes and substantially reduce "scrap loss" not always possible from molded fiberglass grating panels. In addition, since the pultrusion process is automated, it produces a consistent, high quality finished product.

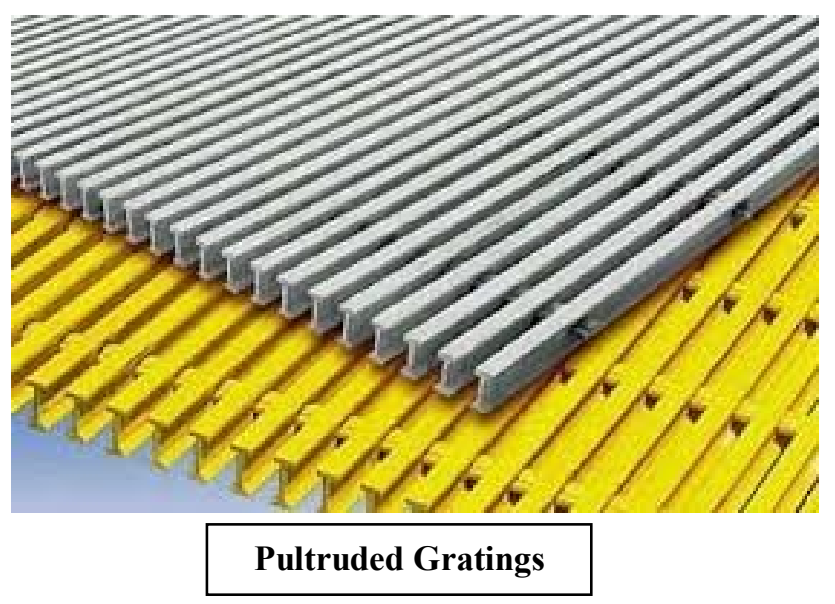

\section{Manufacturing Of Molded Grating By Hand Lay-Up Operation}

A release agent, usually in either wax or liquid form, is applied to the chosen mold. This will allow the finished product to be removed cleanly from the mold. The isopthalic resin [65\%] is mixed with its hardener and applied to the surface. Fiber glass roving [35\%] is laid into the mold, then more resin mixture is added using a brush or roller. The material must conform to the mold, and air must not be trapped between the fiberglass and the mold. Additional resin is applied and possibly additional fiberglass roving.

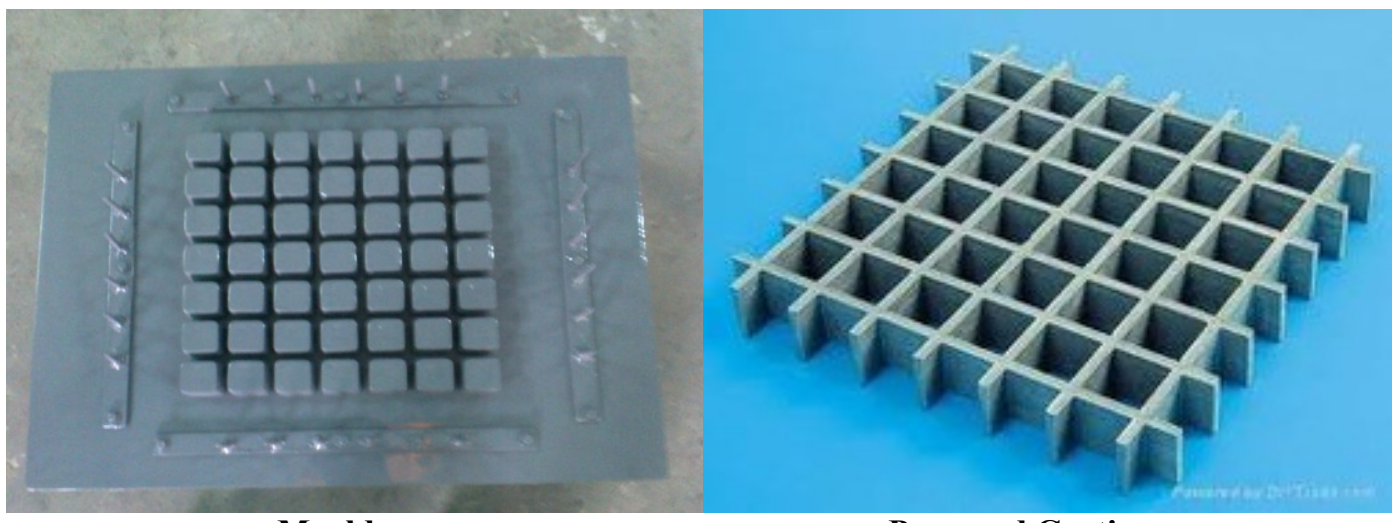

\section{Mould}

Prepared Grating

Hand pressure, vacuum or rollers are used to make sure the resin saturates and fully wets all layers, and any air pockets are removed. The work must be done quickly enough to complete the job before the resin starts to cure, unless high temperature resins are used which will not cure until the part is warmed in a mold. In some cases, the 
work is covered with plastic sheets and vacuum is drawn on the work to remove air bubbles and press the fiberglass to the shape of the mold.

\section{Experimentation And Results}

Tests are carried out on a molded fibre glass grating are:

1. Tensile test 2. Flexural test 3. Chemical resistance test 4 . Load bearing capacity.

\section{Tensile Test:}

Tensile test is carried out on molded fibre glass grating to know the ultimate tensile strength and cross breaking strength. The mechanical properties of materials are determined by performing carefully designed laboratory experiments that replicate as nearly as possible the service conditions. In real life, there are many factors involved in the nature in which loads are applied on a material.

The following are some common examples of modes in which loads might be applied: tensile, compressive, and shear. These properties are important in materials selections for mechanical design. Other factors that often complicate the design process include temperature and time factors.

The topic of this lab is confined to the tensile property of polymers. The above figure shows a tensile testing machine similar to the one used in this lab. This test is a destructive method, in which a specimen of a standard shape and dimensions (prepared according to ASTM D 638: standard test method for tensile properties of plastics) is subjected to an axial load. During a typical tensile experiment, a dog-bone shaped specimen is gripped at its two ends and is pulled to elongate at a determined rate to its breakpoint; a highly ductile polymer may not reach its breakpoint

. The setup of the experiment could be changed to accommodate different types of mechanical testing, according to the ASTM standard (e.g. compression test, etc).

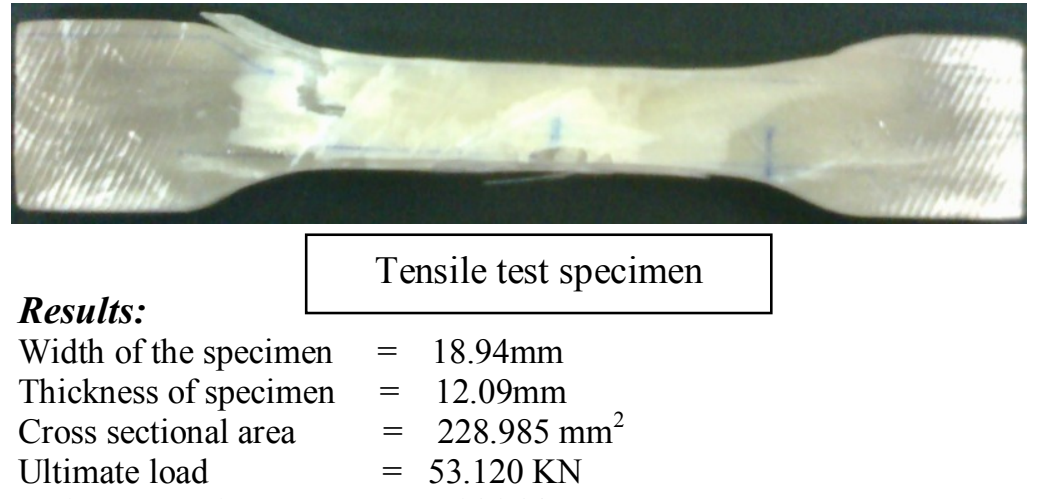

Ultimate tensile strength $=231.985 \mathrm{MPa}$

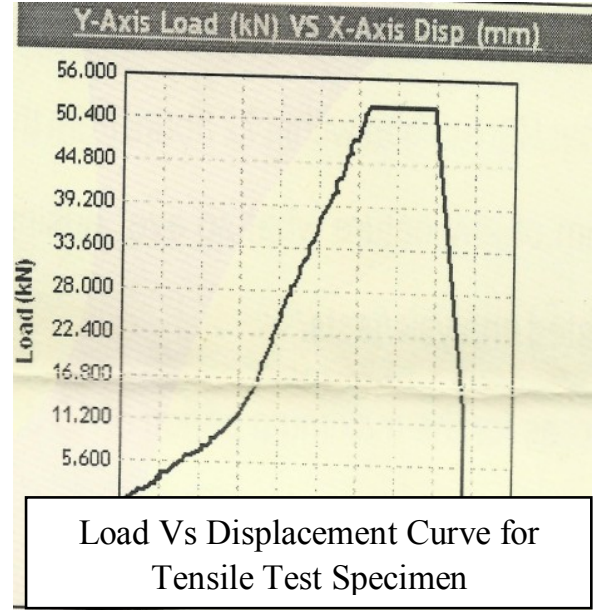

\section{Flexural Test:}

These test methods cover the determination of flexural properties of unreinforced and reinforced plastics, including high-modulus composites and electrical insulating materials in the form of rectangular bars molded directly or cut from sheets, plates, or molded shapes. These test methods are generally applicable to both rigid and semi rigid materials. However, flexural strength cannot be determined for those materials that do not break or that do not fail in the outer surface of the test specimen within the $5.0 \%$ strain limit of these test methods.

This test is a destructive method, in which a specimen of a standard shape and dimensions (prepared according to ASTM D 790-03: standard test method for Flexural strength of plastics) is subjected to a simply supported load.

These test methods utilize a three-point loading system applied to a simply supported beam. A four-point loading system method can be found in Test Method D 6272. Flexural test is carried out on molded fibre glass grating to know the cross breaking strength. Flexural test is carried out as per ASTM D790-03 standard. Their dimensions were determined according to the ASTM D790-03 standard mentioned earlier in the introduction. 


\section{Results:}

Load at fracture $\mathrm{W}$

Distance between supports L

Width of the specimen B

$=9600 \mathrm{~N}$

Thickness of the specimen D

$=100.21 \mathrm{~mm}$

$=21.49 \mathrm{~mm}$

$=12.24 \mathrm{~mm}$

\section{Calculated cross breaking strength $=448.20$}

\section{$\mathbf{N} / \mathbf{m m}^{2}$}

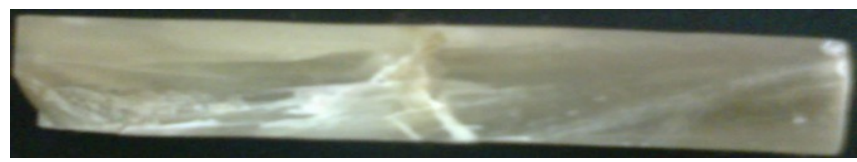

\section{Chemical Resistance Test:}

ASTM D543 - 95, Standard Test Methods for Evaluating Resistance of Plastics to Chemical Reagents, outlines test procedures for determining resistance of the grating to various chemical reagents by means of reporting weight, dimensions, appearance, and strength properties, after a set duration period of immersion. ASTM D543 does not specify the types or concentrations of reagents, duration of the test, or properties to be reported.

The test method consisted of soaking test laminated coupons cut from the glass fibre laminate in the following chemical reagents for a seven day period. For a $100 \mathrm{ml}$ of solution, the $\%$ of chemicals taken was $5 \%$ Caustic Soda, $10 \%$ Sulphuric Acid.

The intial weights of the specimens must be recorded before placing the specimen into the chemical reagents. After seven days period the pieces were taken out from the beakers and rubbed with the soft cloth in order to make the pieces dry. The final weights also recorded. The difference of the two weights can be caluclated to know the performance of the material. The test equipment is as shown below.

Formula for calculating $\%$ of weight loss or gain

$$
\% w=\frac{W f-W i}{W i}
$$

Where $\mathrm{W}_{\mathrm{f}}=$ Finalweight of the specimen ;

$\mathrm{W}_{\mathrm{i}}=$ Intial weight of the specimen

\begin{tabular}{|c|c|c|c|}
\hline Chemicals used & Initial weight & Final weight & \% Change in weight \\
\hline $\mathbf{1 0 \%}$ Sulphuric Acid & 9.555 & 9.565 & 0.18 \\
\hline $\mathbf{5 \%}$ Caustic Soda & 9.232 & 9.243 & 0.119 \\
\hline
\end{tabular}

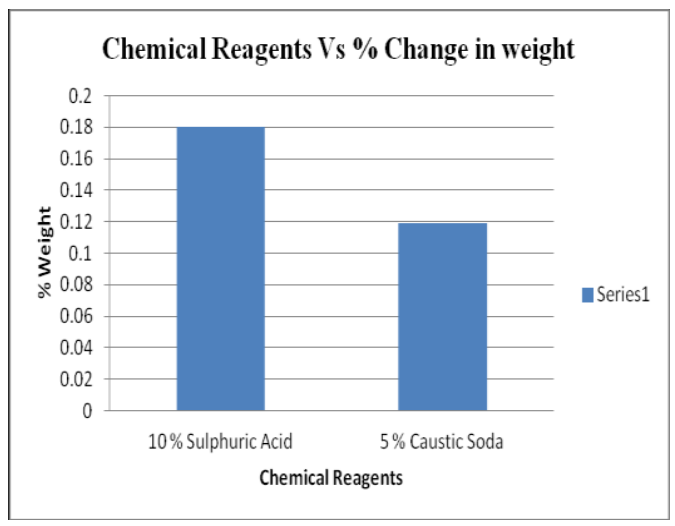

\section{Load Bearing Capacity:}

Molded high load capacity (HLC) grating is yet another product in the arsenal of engineered fiberglass reinforced plastic (FRP) solutions by fiber grate. While capitalizing on most of the traditional benefits of the molded grating products - high strength, corrosion resistance, fire retardancy, non conductivity and low maintence this is specially manufactured by molded as well as pultruded process in FRP composite materials.

There are different types of standard panel sizes with respect to depth, mesh, clear span, and \% of open area will available under ASTM E-84 standard. 


\begin{tabular}{|c|c|c|c|c|c|c|c|c|c|c|c|}
\hline \multicolumn{12}{|c|}{ Fiber grate/Chemgrate Uniform Load Table - Deflection in mm } \\
\hline \multirow{2}{*}{$\begin{array}{c}\text { Clear } \\
\text { Span } \\
\text { mm }\end{array}$} & \multicolumn{2}{|c|}{ Style } & \multicolumn{7}{|c|}{$\operatorname{Load}=\mathrm{KN} / \mathrm{m}^{2}$} & \multirow{2}{*}{$\begin{array}{c}\text { Maximu } \\
\text { m Load } \\
\left(\mathrm{KN} / \mathrm{m}^{2}\right)\end{array}$} & \multirow{2}{*}{$\begin{array}{l}\text { Ultimate } \\
\text { Capacity } \\
\left(\mathrm{KN} / \mathrm{m}^{2}\right)\end{array}$} \\
\hline & $\begin{array}{c}\text { Depth } \\
\text { mm }\end{array}$ & $\begin{array}{c}\text { Mesh } \\
\text { mm }\end{array}$ & 2.50 & 3.00 & 5.00 & 10.00 & 25.00 & $\mathbf{5 0 . 0 0}$ & $\mathbf{1 0 0 . 0 0}$ & & \\
\hline 300 & 38 & $38 \times 38$ & 0.10 & 0.20 & 0.33 & 0.60 & 0.80 & 1.50 & 2.50 & 103.07 & 824.55 \\
\hline
\end{tabular}

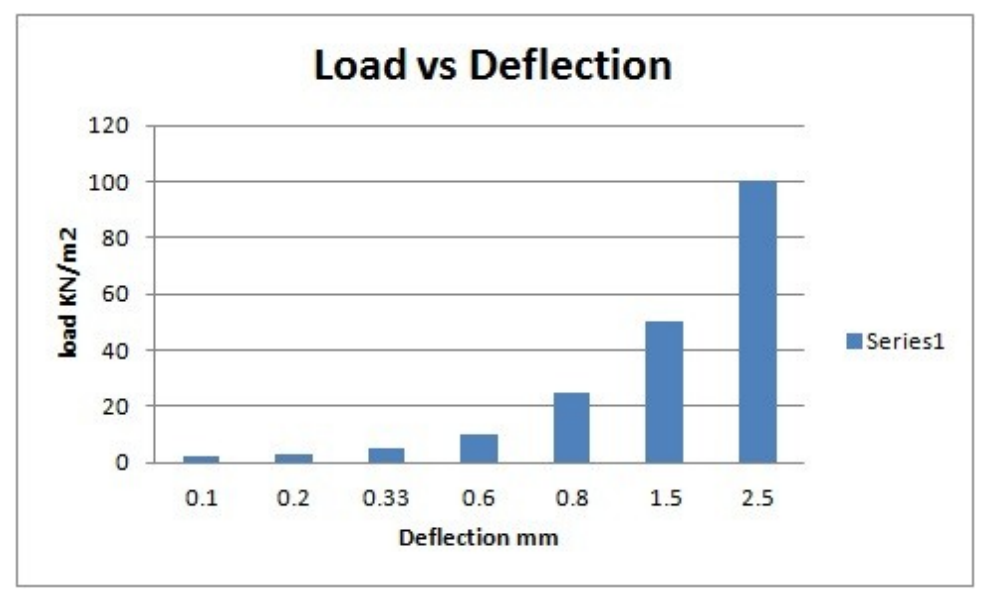

\section{Conclusion}

The Grating is manufactured by combination of resin and fiber glass. It is subjected to Tensile, Flexural, Chemical resistance test and Load bearing test. In chemical resistance test the composite grating is subjected to $10 \%$ sulphuric acid, 5\% caustic soda and can also be tested with another acids and bases. The cost of grating is lowered compared to metal/metal alloy system. Though, the tensile test result is close to mild steel yield strength GFRP grating will be used, because of rust free, durability and maintenance factors.

\section{Acknowledgements}

Authors thank the Head of the department mechanical engineering and Director, Sreenidhi Institute of Science and Technology Hyderabad for extending all sorts of help in bringing out this technical paper.

\section{References}

[1] R.M. Jones, mechanics of composite materials, Mc Graw Hill Company, New York, 1975.

[2] L. R. Calcote, Analysis of laminated composite structures, Van Nostrand Rainfold, New York, 1969.

[3] P. K. Mallik, Fiber Reinforced Composites, $2^{\text {nd }}$ Edition, Marcel Dekker Inc., USA, 1993.

[4] Jones, R.M. (1999) Mechanics of composite materials ( $2^{\text {nd }}$ edition) Phidelphia: Taylor \& Francis. 